\title{
SOLAR MICROWAVE SPIKE EMISSION AND CHANGE OF MAGNETIC TOPOLOGY
}

\author{
FU Qijun \\ Beijing Astronomical Observatory, Chinese Academy of Sciences, \\ Beijing 100080, China. (BAO)
}

GONG Yuanfang, SHANG Qiongzhen, LU Songquan and HU Hanming Yunnan Astronomical Observatory, Chinese Academy of Sciences, Kunming 650011, China. (YAO)

\section{Chunsheng}

Department of Astronomy, Nanjing University, Nanjing 210008, China. (NJU)

LI Wei, ZHAO Bing

Beijing Astronomical Observatory, Chinese Academy of Sciences, Beijing 100080, China. (BAO)

\section{INTRODUCTION}

Electron cyclotron maser (ECM) instability is today's most favoured process for microwave spike emission. Although ECM looks attractive, the emission mechanism is still unclear due to the imperfection of present theories, inadequacy of observational data, and uncertainty as to conditions in the source region. To find solar active phenomena, both statistically and individually coincident with radio spike events, is essential for understanding the ambient conditions needed for generating spike emission, locating sites where spike emission is produced, and providing clues for particle acceleration and energy release in flares.

In this paper, evidence for an association between spike emission at $21 \mathrm{~cm}$ and fast variation of the magnetic configuration in post-flare loops is presented. Such associations may be helpful for solving the questions mentioned above.

\section{QBSERVATIONS}

2.1 A large flare occurred at about 0040-0300 UT on 1989 August 17. It was a solar limb-event, with post-flare loops enduring for more than one day. The flare occurred in AR 5629 , located at $10^{\circ}-13^{\circ}$ behind west limb of solar disk. Using the Solar Magnetic Field Telescope (SMFT) of Huairou Solar Observatory of BAO (Li, W., 1990), it was found with $H_{\beta}$ observations after 0132 UT that a post-flare loop system (PFLS) $10^{4} \mathrm{~km}$ high and $1.8 \times 10^{4} \mathrm{~km}$ wide had formed above the west limb. By 0238 UT the PFLS had reached $6.2 \times 10^{4} \mathrm{~km}$ high. 

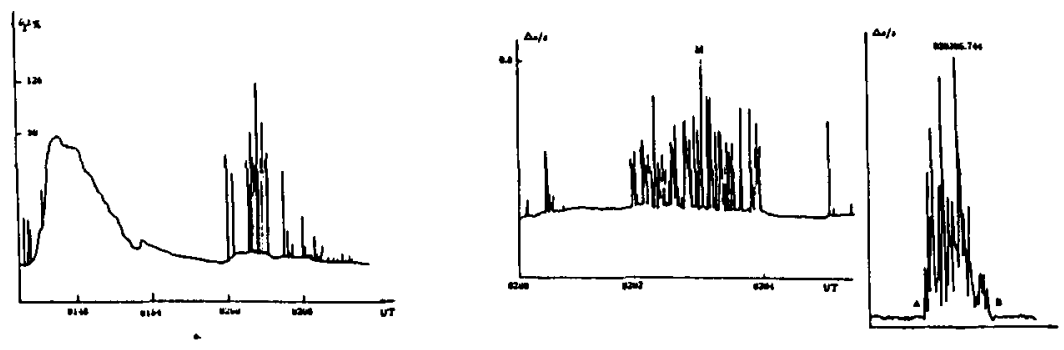

Figure 1. Time profiles of the August 17, 1989 event at $1420 \mathrm{MHz}$. a. time profile in sec time scale; $b$. the compressed profile of msec spike group between 0200-0206 UT; c. the extended profile of the group $M$ indicated in Fig. $1 \mathrm{~b}$. The time interval between $A$ and $B$ is $432 \mathrm{msec}$.

2.2 The $10 \mathrm{~m}$ dish radiotelescope (10MRT) at $21 \mathrm{~cm}$ at YAO has high temporal resolution ( $8 \mathrm{msec}$ ) and high sensitivity $(0.2 \mathrm{s.f.u})$. (Gong, et al., 1989). Figure 1a (Gong, et al., 1990) shows parts of the post-peak and gradual descending phases of the burst. Several fast spike groups were recorded between 0200-0206 UT. Figure 1b shows compressed profiles of the spike groups, and Figure 1c shows extended profiles (with duration of $432 \mathrm{msec}$ ) of the group labelled $\mathrm{M}$ in Figure 1b, which contained the strongest spike in the event, with flux density about 200 s.f.u.. Spikes obviously appear in groups. In the major section of Figure $1 \mathrm{~b}$ between 0202:09-0204:07, a $118 \mathrm{sec}$ time interval, there continually appeared more than 1500 spikes divided into about 40 groups. Most of groups lasted several hundred msec and had 10-30 spikes. The duration of individual spikes was about $20-40 \mathrm{msec}$. The repetition rate was $48 / \mathrm{sec}$ in the most crowded section. The amplitude of spikes was about $50-100 \%$ of $S_{o}$, where $S_{o}=161$ s.f.u. was the $21 \mathrm{~cm}$ quiet solar flux density of this day. The amplitude and duration of the most intense spike were 200 s.f.u. and $30 \mathrm{msec}$.

2.3 At 0132 UT a PFLS composed of at least 11 loops had been formed above the solar west limb. The direction in the photographs are: left-north; right-south; up-west; and down-east. In general the north feet of PFLS are pole $\mathrm{N}$, and south feet are pole S. Figure 3 gives schematic drawings of Figure 2 for clairty, in which some key features are marked. The main $H_{\beta}$ phenomena coincident with the $21 \mathrm{~cm}$ spike groups are as follows:

1) Loop B appeared $5 \mathrm{~min}$ before the occurrence of the spike groups (Fig. 3b);

2) The rising speed of loop $B$ was about $20 \mathrm{~km} / \mathrm{sec}$, faster than that of PFLS. When it caught up with loops of PFLS, the processes of magnetic loop coalescence and reconnection began, causing the brightening of the top of loop $B$ and forming spot $C$ (Fig. $3 \mathrm{c}$ and $3 \mathrm{~d}$ ). Brightening of spot $\mathrm{C}$ began a little before the appearing of radio spike groups;

3) A catastrophic change of magnetic topology occurred between 0200:52-0205:49 UT, causing the disappearance of loop $B$, the appearance of loop $D$, and the top of loop D contacted with PFLS and the bright strip E was formed (Fig. 3d and 3e). This change of magnetic structures was simultaneous with the appearance of spike groups. It is likely that the violent change in magnetic structures, and contact, coalescence and reconnection of magnetic loops caused the spike emission.

\section{DISCUSSION}



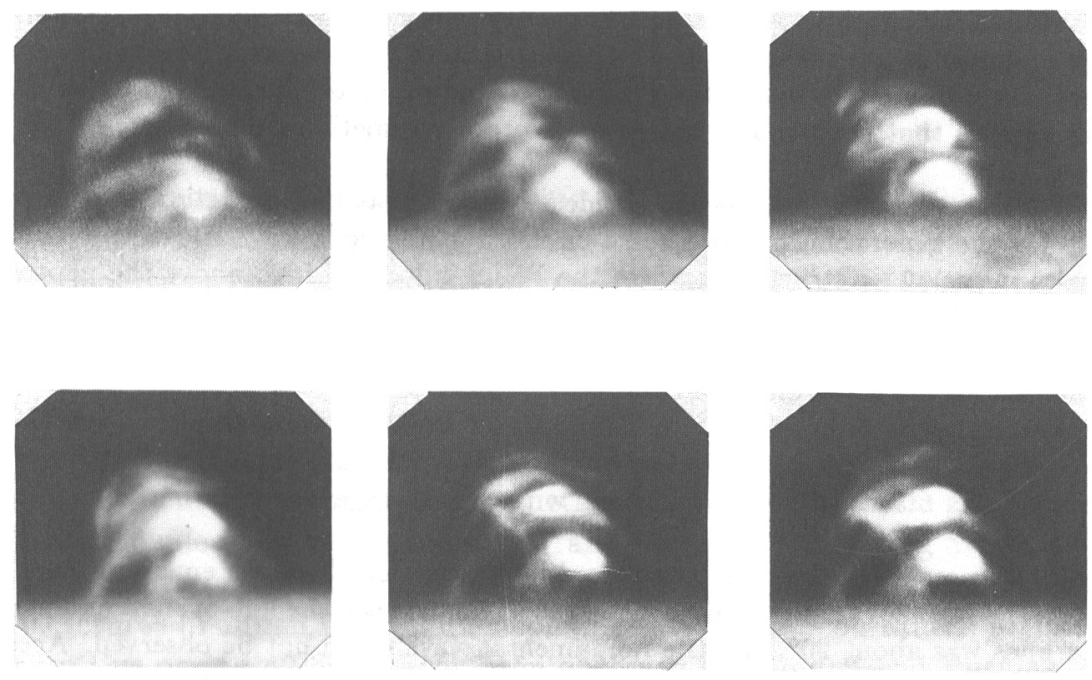

$30 "$

Figure 2. A sequence of $H_{3}$ images coincident to spike groups.



a. 0150:46 UT

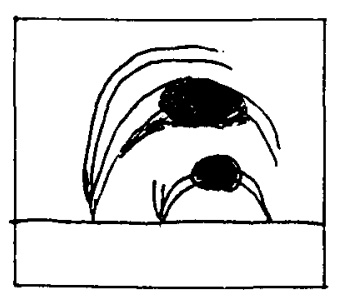

d. 0200:52 UT

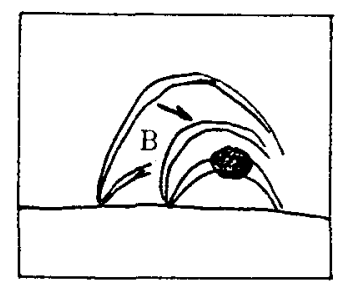

b. 0153:54 UT

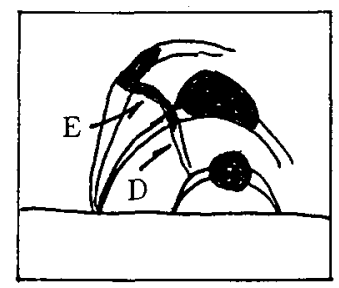

e. 0205:49 UT

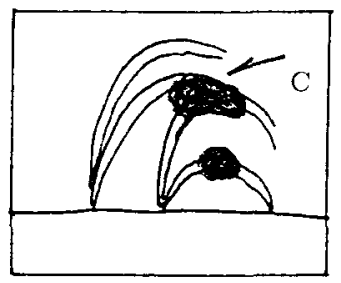

c. 0157:38 UT

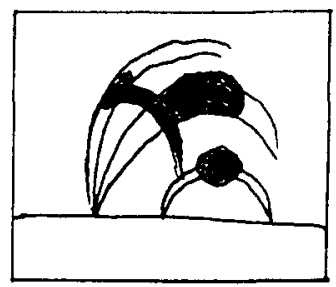

f. 0209:23 UT

Figure 3. Schematic drawing of Figure 2. 
The existence of energetic electrons is seen as a necessary condition for generating spike emission in different theoretical mechanisms. Thus, the appearance of spike emission means that there must exist a process to produce energetic particles. Most spike emission events appear in pre-impulsive and impulsive phases. A number of msec spike emission events have been observed in the gradual descending phase of radio bursts by 10MRT. This implies that the particle accelerating process sometimes takes place in the decay phase.

The event on 1989 August 17 provided evidence that the energetic electrons responsible for the generation of msec spike emission were produced coincident with drastic change in magnetic structures. Since the PFLS is located high above the photosphere $\left(3-4 \times 10^{4} \mathrm{~km}\right.$ in the 1989 August 17 event, It is the layer which electron plasma frequency is in the short $\mathrm{dm}$ waveband) spike emission in the descending phase is liable to happen in the short $\mathrm{dm}$ waveband.

According to Benz (1986), the duration of spikes is comparable to the electronion collision time. In our case, with $\nu=1.42 \mathrm{GHz}$, and taking $T=1.3 \times 10^{5} \mathrm{~K}$, then $\tau=33 \mathrm{msec}$, which is consistent with the average duration of spikes observed, 20-40 msec.

If the msec spike emission was caused by interactions between emerging small loops and the original PFLS on the limb, then the angle between the line-of sight and magnetic field lines was about $90^{\circ}$, and the fundamental wave could not be observed. According to Winglee and Dulk (1986), the emission could be in $x$-mode at the second harmonic.

According to Winglee and Dulk (1986), the fastest growth rate for second harmonic $\mathrm{x}$-mode occurr when $0.6 \leq \omega_{p} / \Omega_{e}<\sqrt{2}$. Assuming $\omega_{p} / \Omega_{e}=1.4$, the magnetic field strength in the source region, $B=180 G$, at the bottom of the loop system and $n_{e}=$ $6.1 \times 10^{9} \mathrm{~cm}^{-3}$, can be deduced.

From deduced $\mathrm{B}$ and $n_{e}$, then the Alfven speed, $V_{a}=5 \times 10^{3} \mathrm{~km} / \mathrm{s}$, can be obtained. Assuming the radiation mechanism propagated with Alfven speed, then the source diameter $l=160 \mathrm{~km}$, can be deduced.

Using deduced $l$ and observed strength of spike emission ( 200 s.f.u.), the brightness temperature of spike source can be estimated

$$
T_{b}=3.6 \times 10^{13} \mathrm{~K} \quad(\text { for } l=160 \mathrm{~km})
$$

\section{ACKNOWLEDGEMENTS}

This work was supported by grant No.9187006-02 of NSFC, No.1870605 of NFSC and No.87-64 of Key Item of Chinese Academy of Sciences.

\section{REFERENCES}

Benz, A.O.: 1986, Solar Phys. 104, 99.

Gong, Y.F., Lu, S.Q., Shang, Q.Z., Hu, H.M., Shi, S.B., and Li, Q.Y.: 1989, Acta Astrophyca Sinia $\underline{9}, 69$. (in Chinese)

Gong, Y.F., Shang, Q.Z., Hu, H.M., Lu, S.Q., Li, J.K., and Yan, R.B.: 1990, Science Bulletin NO.3, 201. (in Chinese)

Li, W.: 1990, Publications of Yunnan Observatory. 22 MAX Global Character Research of Solar-Terrestrial System Special Issue III. 185.

Winglee, R.M. and Dulk, G.A.: 1986, Solar Phys. 104, 93. 